\title{
DYNAMICS OF CYLINDRICAL TURBULENT SPOT IN A LONGITUDINAL SHEAR FLOW OF A PASSIVE STRATIFIED FLUID
}

\author{
Gennadiy G. Chernykh ${ }^{a, b}, *$, Anzhella V. Fomina ${ }^{c}$
}

\begin{abstract}
a Institute of Computational Technologies of SB RAS, Acad Lavrentyev 6, Novosibirsk, 630090 Russian Federation

${ }^{\mathrm{b}}$ Novosibirsk State University, Pirogova Str., 2, Novosibirsk, 630090 Russian Federation ${ }^{c}$ Novokuznetsk Institute (branch) of Kemerovo State University, Tsiolkovskii Str., 23, Novokuznetsk, 654041 Russian Federation
\end{abstract}

Received Septenber 29, 2016;

*e-mail: chernykh@ict.nsc.ru

Accepted in revised form October 22, 2016;

Published December 30, 2016

\begin{abstract}
A numerical model is constructed and dynamics of the cylindrical localized area of turbulent disturbances (turbulent spot) in $a$ longitudinal horizontally homogeneous shear flow of a passive stratified fluid is studied. The results of calculations show a significant turbulent energy generation by shear flo. The problem of flow similarity with respect to the shear Froude number for sufficiently large values of this parameter is considered.
\end{abstract}

Keywords: mathematical model of a turbulent spot in a shear flow, e $\sim \varepsilon$ turbulent model, numerical simulation

DOI: $10.21603 / 2500-1418-2016-1-2-102-107$.

\section{INTRODUCTION}

Evolution of localized regions of turbulent fluid (spots of turbulent) has a decisive influence on formation of a fine microstructure of hydrophysical fields in the ocean [1]. A fairly detailed analysis of studies in the field of the turbulent spot dynamics can be found in [2-4]. The problem of evolution of the turbulent mixing zone in momentumless turbulent wake in a transverse shear flow of a homogeneous fluid is considered in [4]. It is shown that a shear flow may lead to significant deformation of a turbulent area and generate a substantial turbulent energy prolonging the wake lifetime. Flow similarity with respect to the shear Froude number, equal to the ratio of the product of characteristic velocity of turbulent perturbations in the initial time and the characteristic time, caused toby shear flo, to the initial size of the turbulent region is shown $D$. Dynamics of turbulent spot in a transverse linear shear flow of linearly stratified fluid is studied in [5]. It was found that a shear flow in comparison with the case of a homogeneous fluid causes a further significant distortion of the pattern of internal waves generated by the turbulent spot. This paper describes a plane non-stationary problem of dynamics of turbulent disturbances in the longitudinal shear flow of passively stratified fluid. At the initial moment, a turbulent area represents an infinitely long cylinder directed along the axis $x$. Plane section of a cylinder $(y, z)$ is shown in Fig.1; the figure also shows a linear shear flow directed along the axis of the cylinder. A numerical flow model based on the two-parameter semi-empirical turbulent model is built. The results of calculations illustrate the dynamics of turbulent fluid area, accompanied by a significant turbulent energy caused by the effect of a shear flo . The problem of a flow similarity with respect to the shear Froude number is discussed.

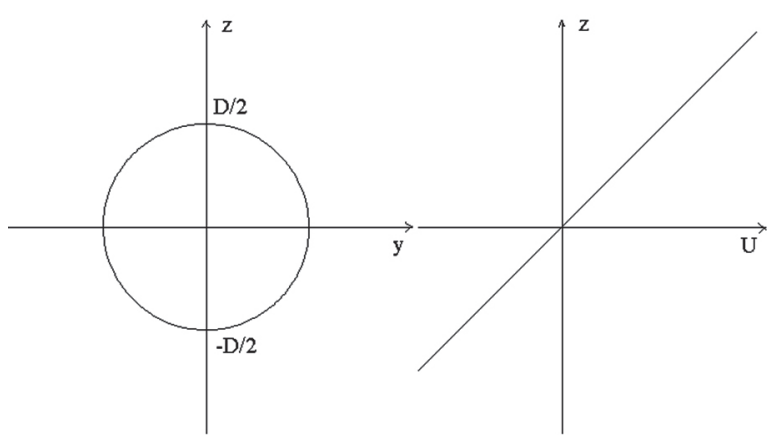

Fig. 1. Flow diagram at the initial moment.

\footnotetext{
Please cite this article in press as: Chernykh G.G., Fomina A.V. Dynamics of cylindrical turbulent spot in a longitudinal shear flow of a passive stratified fluid. Science Evolution,, 2016, vol. 1, no. 2, pp. 102-107. doi:10.21603/2500-1418-2016-1-2-102-107. Copyright (c) 2016, KemSU. This is an open access article distributed under the terms of the Creative Commons Attribution 4.0 International License (http:// creativecommons.org/licenses/by/4.0/), allowing third parties to copy and redistribute the material in any medium or format and to remix, transform, and build upon the material for any purpose, even commercially, provided the original work is properly cited and states its license. This article is published with open access at http://science-evolution.ru/
} 


\section{PROBLEM STATEMENT}

To describe the flo , a mathematical model based on the $e$ model of turbulent is used:

$$
\begin{gathered}
\frac{\partial U}{\partial t}=\frac{\partial}{\partial y} K_{u} \frac{\partial U}{\partial y}+\frac{\partial}{\partial z} K_{u} \frac{\partial U}{\partial z}, \\
\frac{\partial e}{\partial t}=\frac{\partial}{\partial y} K_{e} \frac{\partial e}{\partial y}+\frac{\partial}{\partial z} K_{e} \frac{\partial e}{\partial z}+P-\varepsilon, \\
\frac{\partial \varepsilon}{\partial t}=\frac{\partial}{\partial y} K_{\varepsilon} \frac{\partial \varepsilon}{\partial y}+\frac{\partial}{\partial z} K_{\varepsilon} \frac{\partial \varepsilon}{\partial z}+c_{\varepsilon 1} \frac{\varepsilon}{e} P-c_{\varepsilon 2} \frac{\varepsilon^{2}}{e}, \\
\frac{\partial\left\langle\rho_{1}\right\rangle}{\partial t}=\frac{\partial}{\partial y} K_{\rho} \frac{\partial\left\langle\rho_{1}\right\rangle}{\partial y}+\frac{\partial}{\partial z} K_{\rho} \frac{\partial\left\langle\rho_{1}\right\rangle}{\partial z}+\frac{d \rho_{s}}{d z} \frac{\partial K_{\rho}}{\partial z}, \\
\frac{\partial\left\langle\rho^{\prime 2}\right\rangle}{\partial t}=\frac{\partial}{\partial y} K_{1 \rho} \frac{\partial\left\langle\rho^{\prime 2}\right\rangle}{\partial y}+\frac{\partial}{\partial z} K_{1 \rho} \frac{\partial\left\langle\rho^{\prime 2}\right\rangle}{\partial z}+ \\
+2 K_{\rho}\left[\left(\frac{\partial\langle\rho\rangle}{\partial y}\right)^{2}+\left(\frac{\partial\langle\rho\rangle}{\partial z}\right)^{2}\right]-c_{T} \frac{\left\langle\rho^{\prime 2}\right\rangle \varepsilon}{e} .
\end{gathered}
$$

In equations (1)-(5), the value $U=U(t, y, z)$ is a longitudinal horizontal velocity component; $\left\langle\rho_{1}\right\rangle$ is an averaged density defect: $\left\langle\rho_{1}\right\rangle=\langle\rho\rangle-\rho_{s}$, $\rho_{s}=\rho_{0}(1-a z), a=$ const $>0 ;\left\langle\rho^{\prime 2}\right\rangle$ is a dispersion of the density fluctuations; $e$ is an turbulent energy, $\varepsilon$ is a dissipation rate; $P$ is a turbulent energy generation by gradients of averaged motion; \langle\rangle is a sign of averaging; terms containing factors in the form of molecular viscosity and diffusion coefficie ts were rejected on the assumption of their smallness.

The turbulent viscosity and diffusion coefficients are defined as follows

$$
K_{u}=K_{e}=c_{\mu} \frac{e^{2}}{\varepsilon}, K_{\varepsilon}=\frac{K_{e}}{\sigma}, K_{\rho}=c_{\rho} \frac{e^{2}}{\varepsilon}, K_{\mathrm{l \rho}}=c_{1 \rho} \frac{e^{2}}{\varepsilon} .
$$

Values $c_{\mu}=0.136, \quad \sigma=1.3, \quad c_{\rho}=0.208, \quad c_{1 \rho}=0.087$, $c_{T}=1.25, \quad c_{\varepsilon 1}=1.44, \quad c_{\varepsilon 2}=1.92$ are known empirical constants $[6,7]$.

For $t=t_{0}$, the following initial conditions were specified

$$
\begin{gathered}
e\left(t_{0}, y, z\right)=\Phi_{1}(r), \varepsilon\left(t_{0}, y, z\right)=\Phi_{2}(r), \\
U\left(t_{0}, y, z\right)=\alpha z \sqrt{e_{0}} / D=U_{s}(z),\left\langle\rho_{1}\right\rangle=\left\langle\rho^{\prime 2}\right\rangle=0, \\
-\infty<y<\infty,-\infty<z<\infty, t=t_{0} .
\end{gathered}
$$

Here, $\alpha=$ const, $D-$ is a diameter of turbulent mixing zone in the initial moment $e_{0}=e\left(t_{0}, 0,0\right)$. Functions $\Phi_{1}(r), \Phi_{2}(r), r^{2}=y^{2}+z^{2}$ are finite bellshaped functions, consistent with the experimental data of Lin and Pao (Lin, Pao) on decay of momentumless turbulent wake in a homogeneous fluid [8]. For $r^{2}=y^{2}+z^{2} \rightarrow \infty$ the following conditions $e=\varepsilon=\left\langle\rho_{1}\right\rangle=\left\langle\rho^{\prime 2}\right\rangle=0, \quad U=U_{s}(z), \quad t \geq t_{0} \quad$ were established.
In the calculations the boundary conditions corresponding to $r \rightarrow \infty$, were shifted to borders of a sufficiently large rectangle whose dimensions were chosen based on the results of numerical experiments. For reasons of symmetry the solution is sought in the first quadrant of the plane $(y, z)$. The boundary conditions on the coordinate axes were taken as follows:

$$
\begin{gathered}
\frac{\partial U}{\partial y}=\frac{\partial\left\langle\rho_{1}\right\rangle}{\partial y}=\frac{\partial e}{\partial y}=\frac{\partial \varepsilon}{\partial y}=\frac{\partial\left\langle\rho^{\prime 2}\right\rangle}{\partial y}=0, \quad y=0, z \geq 0 \\
U=\left\langle\rho_{1}\right\rangle=\frac{\partial e}{\partial z}=\frac{\partial \varepsilon}{\partial z}=\frac{\partial\left\langle\rho^{\prime 2}\right\rangle}{\partial z}=0, \quad z=0, y \geq 0 .
\end{gathered}
$$

Variables of the problem can be nondimensionalized with the use of characteristic scales of length $\mathrm{D}$, velocity $U_{0}=\sqrt{e_{0}}$ and density $a D \rho_{0}$. With this nondimensionalization, a characteristic flow time is a quantity $T_{c}=D / \sqrt{e_{0}}$. In such a case, the form of equations (1)-(5) does not change; in the future, if necessary, dimensionless quantities will be designated as*. The problem has another characteristic time $T_{s}=\left(d U_{s} / d z\right)^{-1}=\left(\alpha \sqrt{e_{0}} / D\right)^{-1}$. By analogy with the well-known density Froude number; let us introduce a shear Froude number $F_{s}=\sqrt{e_{0}} T_{s} / D=\alpha^{-1}$ (see also [4]). In this case, $U_{s}^{*}=z^{*} / F_{s}$. is valid. In equations (2), (3), as mentioned above, the quantity $P$ - is an energy, generated by turbulent gradients of the averaged flow

$$
\begin{gathered}
P=-\left\langle u^{\prime} v^{\prime}\right\rangle \frac{\partial U}{\partial y}-\left\langle u^{\prime} w^{\prime}\right\rangle \frac{\partial U}{\partial z}= \\
=K_{u}\left[\left(\frac{\partial U}{\partial y}\right)^{2}+\left(\frac{\partial U}{\partial z}\right)^{2}\right]= \\
=K_{u}\left[\left(\frac{\partial U_{d}}{\partial u}\right)^{2}+\left(\frac{\partial U_{d}}{\partial z}+\frac{d U_{s}}{d z}\right)^{2}\right], U_{d}=U-U_{s} .
\end{gathered}
$$

On the assumption of the concept of dimensionless quantities $U_{s}=U_{s}^{*} \sqrt{e_{0}}, \quad z=z^{*} D$, we obtain

$$
\frac{d U_{s}}{d z}=\frac{\sqrt{e_{0}}}{D} \frac{d U_{s}^{*}}{d z^{*}}=\frac{\sqrt{e_{0}}}{D} \frac{1}{F_{s}} \Rightarrow \frac{d U_{s}^{*}}{d z^{*}}=\frac{1}{F_{s}} .
$$

Thus, dimensionless equations (2), (3) (as well as in (1)) have a quantity $1 / F_{s}$, characterizing presence of a background linear shear longitudinal flow in the problem.

\section{FINITE-DIFFERENCE SOLUTION} ALGORITHM

Finite-difference solution algorithm is based on the consistent time integration of the differential equation system (1) - (5) in each layer. Let us give an example of the finite-di ference analogue of equation (2) based on an implicit splitting scheme $[7,9]$

$$
\frac{e_{i, j}^{n+1 / 2}-e_{i, j}^{n}}{\Delta t}=\frac{\left(K_{e}^{n}\right)_{i+1 / 2, j}\left(e_{i+1, j}^{n+1 / 2}-e_{i, j}^{n+1 / 2}\right)-\left(K_{e}^{n}\right)_{i-1 / 2, j}\left(e_{i, j}^{n+1 / 2}-e_{i-1, j}^{n+1 / 2}\right)}{h_{y}^{2}}+P_{i, j}-\varepsilon_{i, j},
$$




$$
\begin{gathered}
\frac{e_{i, j}^{n+1}-e_{i, j}^{n+1 / 2}}{\Delta t}=\frac{\left(K_{e}^{n}\right)_{i, j+1 / 2}\left(e_{i, j+1}^{n+1}-e_{i, j}^{n+1}\right)-\left(K_{e}^{n}\right)_{i, j-1 / 2}\left(e_{i, j}^{n+1}-e_{i, j-1}^{n+1}\right)}{h_{z}^{2}}, \\
\left(K_{e}^{n}\right)_{i \pm 1 / 2, j}=0.5\left(\left(K_{e}^{n}\right)_{i \pm 1, j}+\left(K_{e}^{n}\right)_{i, j}\right),\left(K_{e}^{n}\right)_{i, j \pm 1 / 2}=0.5\left(\left(K_{e}^{n}\right)_{i, j \pm 1}+\left(K_{e}^{n}\right)_{i, j}\right), \\
e_{i, j}^{n+1}=e\left(t_{0}+(n+1) \Delta t, i \times h_{y}, j \times h_{z}\right),\left(i=0, \ldots, N_{y} ; j=0, \ldots, N_{z} ; n=0, \ldots, N_{t}\right) .
\end{gathered}
$$

$\Delta t, h_{y}, h_{z}$ - time and space variable pitches of a uniform difference grid.

Finite difference equations are solved by the sweep method. The numerical model was tested by comparing the results of calculations on a sequence of grids with numerical calculations of the problem of a wake behind self-propelled and towed bodies in a homogeneous liquid in a one-dimensional formulation and by comparing the calculation results in detail with the experimental data $[7,10]$. Calculations of this work were carried out on a uniform difference grid with the following parameters. $\Delta t^{*}=0.1, \quad h_{y}^{*}=h_{z}^{*}=0.02$. Grid area dimensions $0 \leq y^{*} \leq Y^{*}, \quad 0 \leq z^{*} \leq Z^{*}, \quad Y^{*}=Z^{*}=8$. 4-fold increase of the time pitch while halving $h_{y}, h_{z}$ led to deviations in values of grid solutions, not exceeding $1 \%$ of the uniform norm.

\section{RESULTS OF CALCULATION OF THE TURBULENT SPOT DYNAMICS IN THE LONGITUDINAL LINEAR SHEAR FLOW}

Before presenting the results of calculations, following [4, 11], let us give the considerations concerning similarity with respect to the shear Froude number $F_{s}=\frac{U_{0} T_{s}}{D}=\frac{1}{\alpha}, T_{s}=\left(\frac{d U_{s}}{d z}\right)^{-1}$. It is well known $[2,3,11]$ that in the absence of shear $U_{s}(z)=0$ at a sufficiently long decay times, a flow generated at the evolution of a plane localized area of turbulent disturbances in homogeneous and passive stratified fluid becomes self-similar. In this regard, the following representations are valid:

$$
\begin{gathered}
e=e_{m} f_{1}(r / L), \varepsilon=\varepsilon_{m} f_{2}(r / L), \\
\left\langle\rho_{1}\right\rangle=\left\langle\rho_{1}\right\rangle_{m} H_{1}(y / L, z / L), \\
\left\langle\rho^{\prime 2}\right\rangle=\left\langle\rho^{\prime 2}\right\rangle_{m} H_{2}(y / L, z / L),
\end{gathered}
$$

where $r=\sqrt{y^{2}+z^{2}}, \mathrm{~L}-$ is still the characteristic size of the turbulent spot. In accordance with the results of numerical experiments and analytical studies [7], the following representations are valid for values $e_{m}, \varepsilon_{m},\left\langle\rho_{1}\right\rangle_{m},\left\langle\rho^{\prime 2}\right\rangle_{m}$

$$
\begin{gathered}
\frac{e_{m}}{U_{0}^{2}}=B_{1}\left(\frac{t-t_{0}}{T_{c}}\right)^{-1.54}, \quad \frac{\varepsilon_{m} D}{U_{0}^{3}}=B_{2}\left(\frac{t-t_{0}}{T_{c}}\right)^{-2.54}, \\
\frac{1}{a D \rho_{0}}\left\langle\rho_{1}\right\rangle_{m}=B_{3}\left(\frac{t-t_{0}}{T_{c}}\right)^{0.23}, \\
\frac{1}{\left(a D \rho_{0}\right)^{2}}\left\langle\rho^{\prime 2}\right\rangle_{m}=B_{4}\left(\frac{t-t_{0}}{T_{c}}\right)^{0.46}, \\
\frac{L}{D}=B_{5}\left(\frac{t-t_{0}}{T_{c}}\right)^{0.23} .
\end{gathered}
$$

By analogy with [4] in the case of a linear shear flow $U_{s}=U_{0} \alpha \frac{z}{D}, t \gg 1, F_{s} \gg 1$ solution should be sought in the form $\left(t / T_{c}=\left(t / T_{s}\right) T_{s} / T_{c}=\left(t / T_{s}\right) F_{s}\right)$

$$
\begin{gathered}
\frac{e}{U_{0}^{2}} F_{s}^{1.54}=G_{e}\left(\frac{t}{T_{s}}, \frac{y}{D F_{s}^{0.23}}, \frac{z}{D F_{s}^{0.23}}\right), \\
\frac{\varepsilon D}{U_{0}^{3}} F_{s}^{2.54}=G_{\varepsilon}\left(\frac{t}{T_{s}}, \frac{y}{D F_{s}^{0.23}}, \frac{z}{D F_{s}^{0.23}}\right), \\
\frac{\left\langle\rho_{1}\right\rangle}{a D \rho_{0}} F_{s}^{0.23}=G_{1 \rho}\left(\frac{t}{T_{s}}, \frac{y}{D F_{s}^{0.23}}, \frac{z}{D F_{s}^{0.23}}\right), \\
\frac{\left\langle\rho^{\prime 2}\right\rangle}{\left(a D \rho_{0}\right)^{2}} F_{s}^{0.46}=G_{2 \rho}\left(\frac{t}{T_{s}}, \frac{y}{D F_{s}^{0.23}}, \frac{z}{D F_{s}^{0.23}}\right), \\
\frac{e_{m}}{U_{0}^{2}} F_{s}^{1.54}=\varphi_{e}\left(\frac{t}{T_{s}}\right), \\
\frac{L_{y}}{D} F_{s}^{-0.23}=\varphi_{1 L}\left(\frac{t}{T_{s}}\right), \frac{L_{z}}{D} F_{s}^{-0.23}=\varphi_{2 L}\left(\frac{t}{T_{s}}\right) .
\end{gathered}
$$

Flow generated at the evolution of a localized turbulent area in a longitudinal shear flow is illustrated by change in the characteristic dimensions $L_{y}, L_{z}$, determined from ratios $e\left(t, L_{y}, 0\right)=e(t, 0,0) / 2$, $e\left(t, 0, L_{z}\right)=e(t, 0,0) / 2$. Fig. 2 (a), (b) show graphs of functions $L_{y}(t), L_{z}(t)$ for various $U_{s}(z)$. It can be seen that up to values $t / T_{c} \leq 300$, dimensions are the same for all the above shears $U_{s}(z)$ and a shear-free flo . For large time values the flow is characterized by more intense expansion of a turbulent spot, caused by generation of the turbulent energy in the spot due to a shear flo . Given considerations of similarity with respect to the shear Froude number $F_{s}$, Fig. 2 (c), (d) shows characteristic "universal" curves $F_{s}^{-0.23} L_{y}\left(t / T_{s}\right), \quad F_{s}^{-0.23} L_{z}\left(t / T_{s}\right)$. A certain discrepancy between the results of calculations in the initial interval of dynamics of a turbulent spot is caused by a flow non-self-similarity at the initial time inter in shearless case occurs more later).

Flow is also characterized by behavior of characteristic scale of a turbulent energy $e(t, 0,0)$ depending on time and shear flow $U_{s}(z)$ (Fig. 3 (a)). It can be seen that up to the time $t / T_{c} \leq 400$, values $e(t, 0,0)$ are practically the same (maximum deviation corresponds to $\alpha=0.004$ and is approximately $7 \%$ ); for large time values the flow is characterized by generation of the turbulent energy due to gradients of a longitudinal velocity component $U(t, y, z)$. The total turbulent energy $E_{t}(t)=\int_{0}^{\infty} \int_{0}^{\infty} e d y d z$ behaves similarly (Fig. $3(\mathrm{~b})$ ). The results of the corresponding simulation for the shear 
Froude number $F_{s}$ are shown in Fig. 3 (c), (d). A certain discrepancy between the results of calculations in the

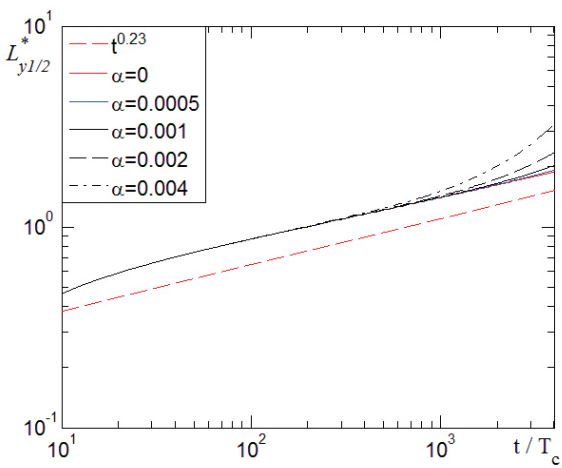

(a)

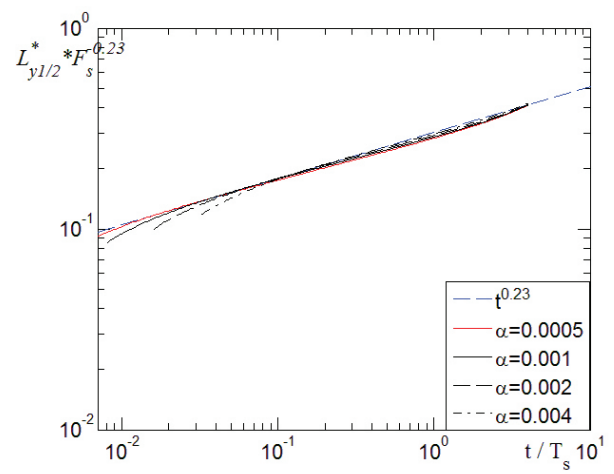

(c) initial stage of decay is also caused by a flow non-selfsimilarity in this time interval.

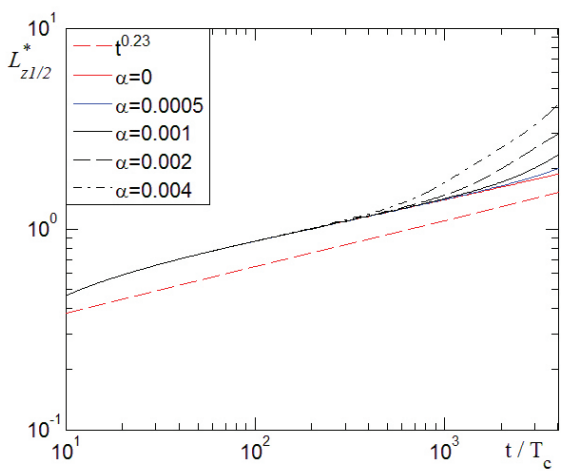

(b)

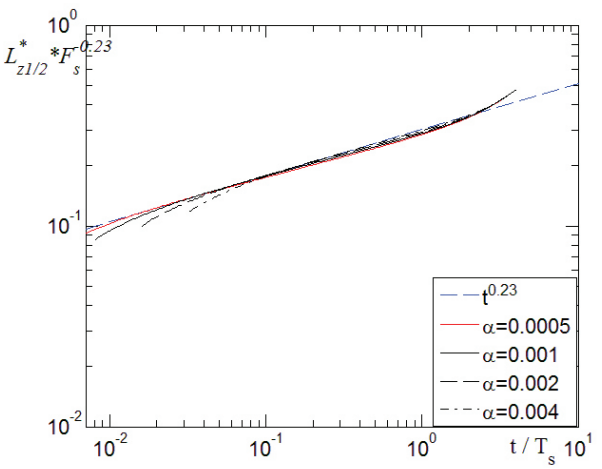

(d)

Fig. 2. Graphs of functions $L_{y}(t)$ - (a); $L_{z}(t)$ - (b); $F_{s}^{-0.23} L_{y}\left(t / T_{s}\right)$ - (c); $F_{s}^{-0.23} L_{z}\left(t / T_{s}\right)$ - (d) for various values $U_{S}(z)$.

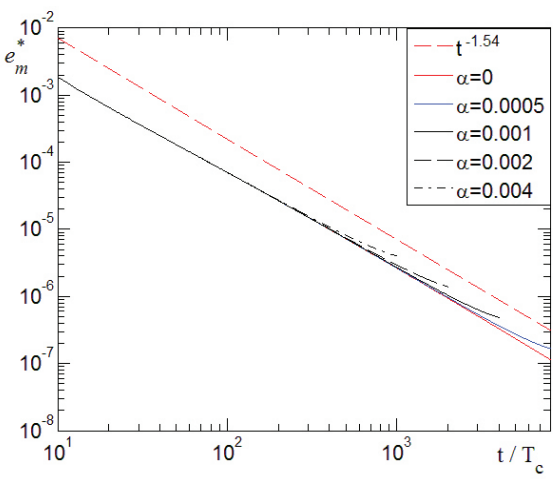

(a)

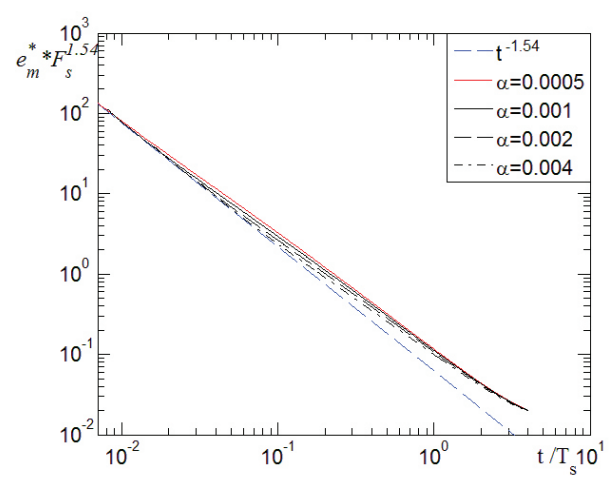

(c)

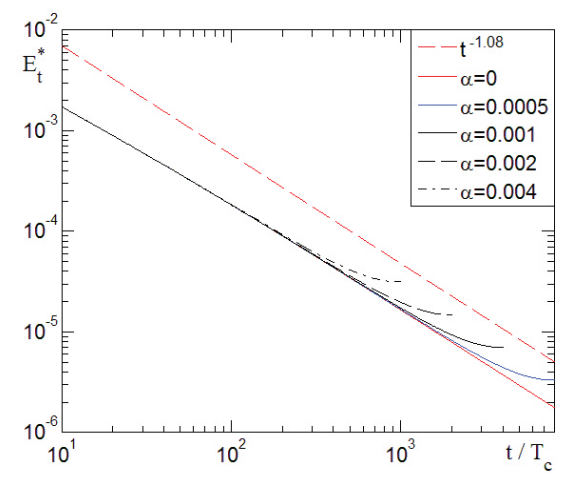

(b)

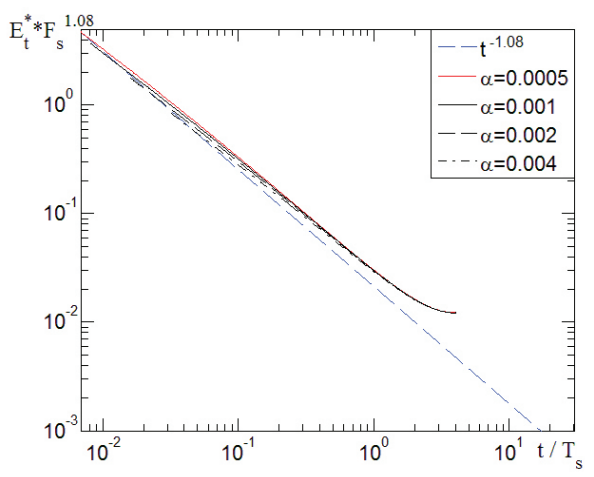

(d)

Fig. 3. Graphs of functions $e_{m}(t)=e(t, 0,0)-(\mathrm{a}) ; E_{t}(t)-(\mathrm{b}) ; F_{s}^{1.54} e_{m}\left(t / T_{s}\right)-(\mathrm{c}) ; F_{s}^{1.08} E_{t}\left(t / T_{s}\right)-(\mathrm{d})$ for various values of $U_{s}(z)$. 
To illustrate similarity with respect to the shear Froude number, Fig. 4 (a), (b), (c), (d), (e), (f) show also graphs of a turbulent energy, a defect density and a dispersion of density fluctuations along the vertical axis in the initial coordinates and flow area "similarity" coordinates for $t / T_{s}=2$. It can be seen that with $F_{s} \geq 1000$ graphs of functions do not differ much from each other, which also indicates the approximate similarity of flow at large $F_{s}$. Appropriate hydrodynamic fields are more fully illustrated by contour lines (Figure 5), corresponding to $t / T_{s}=2$. They were obtained for $\alpha=0.001$.

The main results are as follows. The mathematical model is built and numerical simulation of evolution

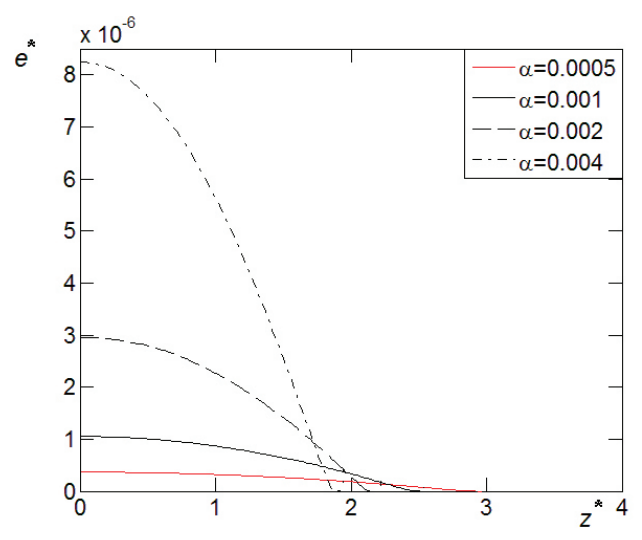

(a)

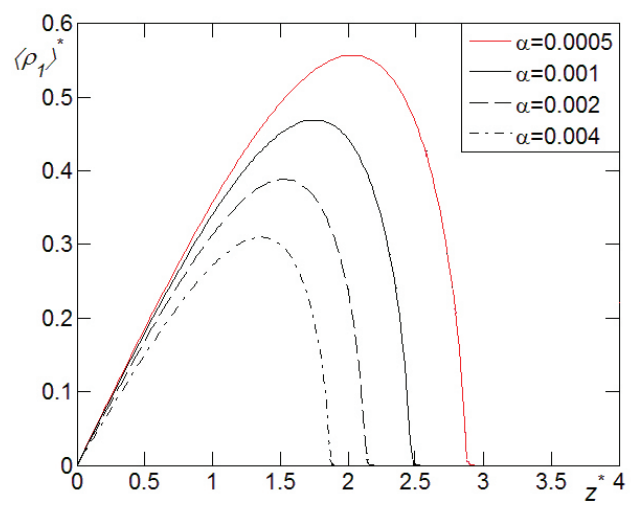

(c)

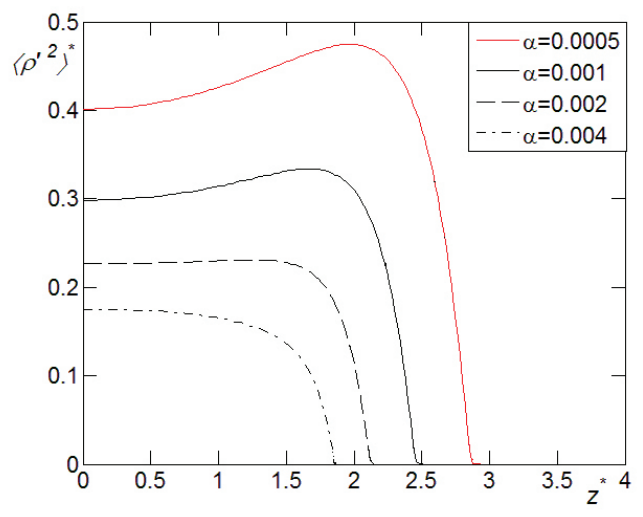

(e) of a cylindrical localized area of turbulent disturbances in a longitudinal horizontally homogeneous shear flow of a passive stratified fluid is carried out. The results of numerical experiment show significant generation of turbulent energy caused by a shear flo . The latter may extend the turbulent spot life. The problem of flow similarity with respect to the shear Froude number for sufficiently la ge values of this parameter is considered. Numerical experiments confirm a flow similarity at large values of the Froude number.

The study was supported by a grant of Leading Scientific Schools NSH - 7214.2016.9 and Russian Fundamental Research Fund (project 17-01-00332).

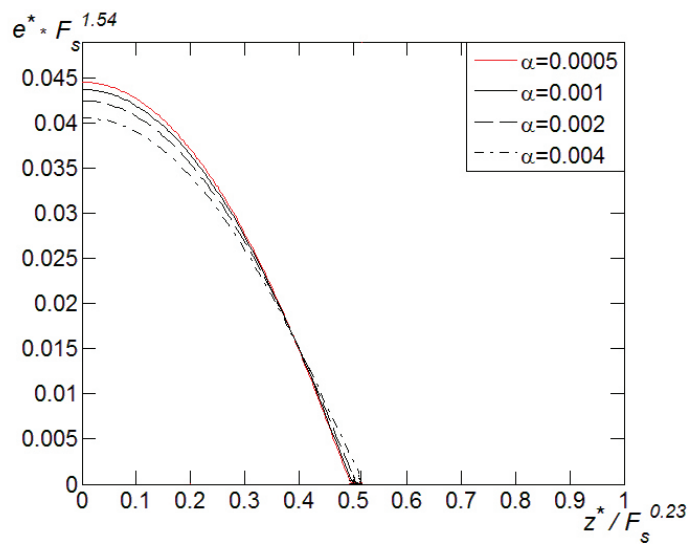

(b)

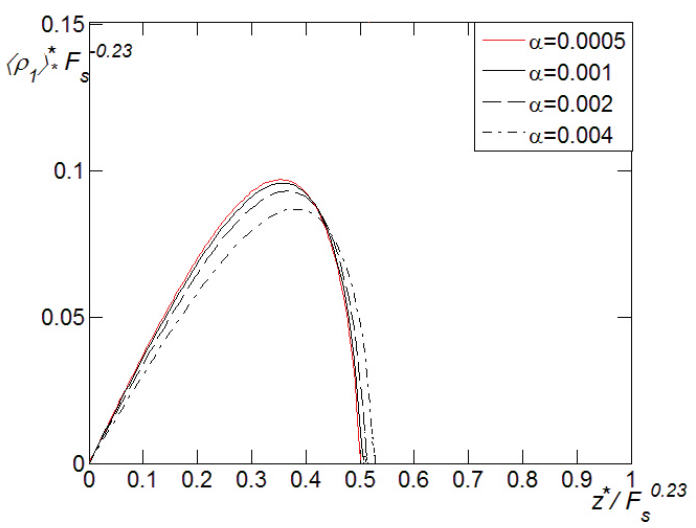

(d)

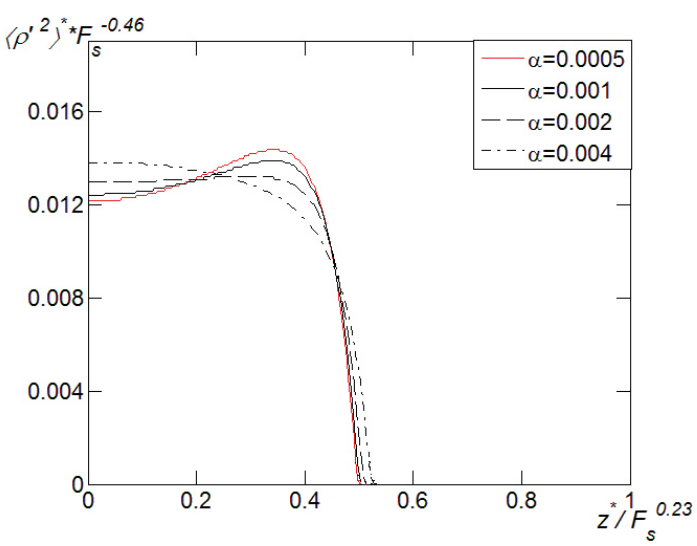

(f)

Fig. 4. Graphs of turbulent energy functions $e$, a density defect $\rho^{\prime 2}$ and a dispersion of density fluctuations $\rho^{\prime 2}$ along the vertical axis in the initial coordinates - (a), (c), (e) and flow area in "similarity" coordinates - (b), (d), (f) for $t / T 2=$ 

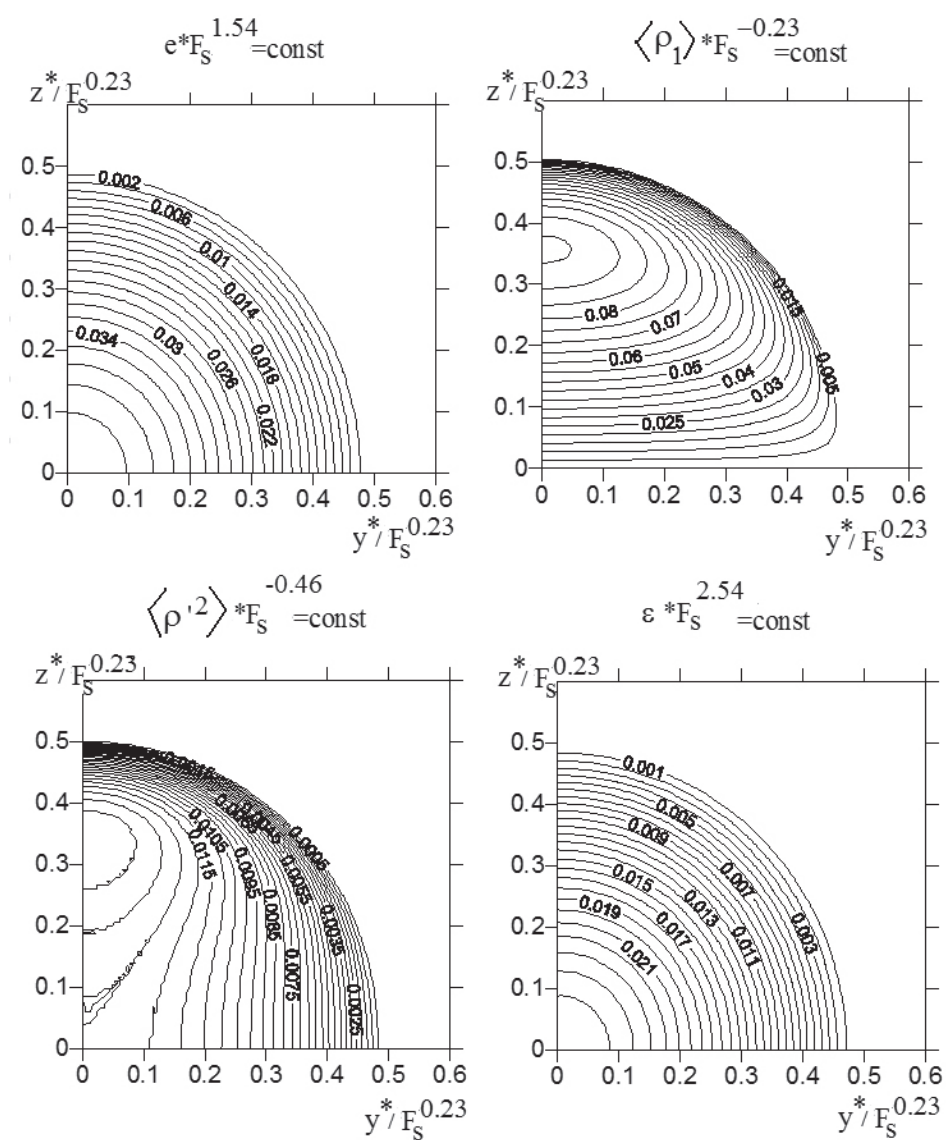

Fig. 5. Universal contour lines of turbulent energy, density defect, dispersion of density fluctuations and dissipation rate, $t / T_{s}=2$.

\section{REFERENCES}

1. Monin A.S., Yaglom A.M. Statisticheskaya gidromekhanika (teoriya turbulentnosti) [Statistical Fluid Mechanics (theory of turbulent )]. St. Petersburg: Gidrometeoizdat, 1992. 696 p.

2. Vasiliev O.F., Kuznetsov B.G., Lytkin Yu.M., Chernykh G.G. Development of the region of a turbulized liquid in a stratified medium. Fluid Dynamics, 1974, vol. 9, no. 3, pp. 368-373.

3. Chashechkin Yu.D., Chernykh G.G., Voropayeva O.F. The propagation of a Passive Admixture from a Local Instantaneous Source in a Turbulent Mixing Zone. IJCFD, 2005, vol. 19, no. 7, pp. 517-529.

4. Chernykh G.G., Voropayeva O.F. Dynamics of a Momentumless Turbulent Wake in a Shear Flow. Journal of Engineering Thermophysics, 2015, vol. 24, no. 1, pp. 12-21.

5. Voropayeva O.F., Chernykh G.G. Dinamika lokal'nykh oblastey turbulizovannoy zhidkosti v usloviyakh fonovykh vozmushcheniy gidrofizicheskikh poley [The Dynamics of Local Zones of Turbulized Fluid under the Background Disturbances of Hydrophysical Fields]. Fundamental'naya i prikladnaya gidrofizika [Fundamental and Applied Hydrophysics], 2015, vol. 8, no. 4, pp. $12-17$.

6. Rodi W. Turbulent models and their application in hydraulics. A state of the art review. University of Karlsruhe, $1980.104 \mathrm{p}$.

7. Kaptsov O.V., Fomina A.V., Chernykh G.G., Shmidt A.V. Self-similar decay of the momentumless turbulent wake in a passive stratified medium. Matem. Model., 2015, vol. 27, no. 1, pp. 84-98.

8. Hassid S. Collapse of turbulent wakes in stable stratified media. Journal of Hydronautics, 1980, vol. 14, no. 1, pp. $25-32$.

9. Samarskii A.A., Vvedenie v teoriyu raznostnykh skhem [Introduction to the Theory of Difference Schemes], Moscow: Nauka Publ., 1971 (in Russian).

10. Chernykh G.G., Fomina A.V., Moshkin N.P. Numerical Simulation of Dynamics of Turbulent Wakes behind Towed Bodies in Linearly Stratified Media. Journal of Engineering Thermophysics, 2009, vol. 18, no. 4, pp. 279-305.

11. Lytkin Yu.M., Chernykh G.G. Similarity of the flow with respect to the density Froude number and energy balance at the evolution of the turbulent mixing zone in a stratified medium. Mathematical Problems of Continuum Mechanics: a Collection of Scientific Works, 1980, no. 47, pp. 70-89.

\section{Gennadiy G. Chernykh}

Dr.Sci.(Phys.-Math.), Professor, Chief Researcher of the Institute of Computational Technologies SB RAS, Novosibirsk, Russian Federation

\section{Anzhella V. Fomina}

Cand.Sci.(Phys.-Math.), Associate Professor, Novokuznetsk Institute (branch) of Kemerovo State University, Novokuznetsk, Russian Federation 